\title{
Factors associated with respiration induced variability in cerebral blood flow velocity
}

\author{
Heather Coughtrey, Janet M Rennie, David H Evans, Tim J Cole
}

\begin{abstract}
A consecutive cohort of 73 very low birthweight infants was studied to determine the presence or absence of beat to beat variability in the velocity of blood flow in the cerebral circulation and its relation with respiration. One minute epochs of information included recordings of cerebral blood flow velocity estimated with Doppler ultrasound, blood pressure, spontaneous respiratory activity, and ventilator cycling. Fourier transformation was used to resolve the frequencies present within the one minute epochs and to classify the cerebral blood flow velocity as showing the presence or absence of any respiratory associated variability. A total of 249 recordings was made on days 1,2 , 3 , and 7. Forty seven infants showed respiratory variability in cerebral blood flow velocity on 97 occasions, usually during the first day of life. The infants with respiratory associated variability were of lower gestational age and when the respiratory associated variability was present they were more likely to be ventilated and receiving higher inspired oxygen; these associations were shown to be independent of gestational age. There was no significant independent association with brain injury, cerebral blood flow velocity $(\mathrm{cm} / \mathrm{s})$, or blood pressure $(\mathrm{mm} \mathrm{Hg})$. The findings suggest that artificial ventilation may entrain normal respiratory associated variability in the cerebral circulation but do not provide evidence that it is harmful.
\end{abstract}

(Arch Dis Child 1993;68:312-316)

Neonatal Intensive

Care Unit,

Rosie Maternity

Hospital, Cambridge

H Coughtrey

J M Rennie

Department of

Medical Physics,

University of Leicester

D H Evans

Department of

Statistics,

MRC Dunn Nutrition

Unit, Cambridge

T J Cole

Correspondence to:

Dr J M Rennie,

Dr J M Rennie,
NICU, Rosie Maternity

Hospital, Robinson Way,

Hospital, Robinson Way,

Accepted 28 October 1992

The cerebral blood flow velocity of preterm infants has been shown to vary beat by beat and minute by minute. A high variability shown with Doppler ultrasound in 23 of 50 ventilated infants was associated with subsequent intracranial haemorrhage in $21 .^{1}$ The explanation which has been proposed for the origin of the high variability, which has been confirmed by several workers, ${ }^{2} 3$ is that the changes are similar to those of pulsus paradoxus in the systemic circulation. The influence of respiration on blood pressure was first recognised in 1733 by Hales. ${ }^{4}$ In the 19th century Traube ${ }^{5}$ and Hering ${ }^{6}$ described fluctuations in blood pressure occurring at the same frequency as respiration in deteriorating animal preparations. Respiratory modulation of heart rate has also been extensively studied in relation to sudden infant death syndrome. ${ }^{7}$
Most previous work which has reported cerebral blood flow velocity variability has relied on measuring the coefficient of variation of the area under the curve of instantaneous peak velocity produced from Doppler ultrasound instrumentation. The mathematical technique of Fourier transformation spectral analysis allows identification of the frequency components of a complex varying signal, rather like working out the individual notes contained in a musical chord. The frequency components of blood pressure or heart rate variation which can be shown using Fourier transformation include the respiratory frequency $(0.5-1.5 \mathrm{~Hz}$ in the newborn infant), the heart rate frequency $(1 \cdot 5-3 \mathrm{~Hz})$, and a low frequency element $(0 \cdot 01-0 \cdot 15 \mathrm{~Hz})$ thought to be influenced by thermoregulation and baroreceptor modulation. ${ }^{8}$

This method of signal analysis has been applied to cerebral blood flow velocity in the newborn infant by Bignall et al, who found that a variation corresponding to the respiratory frequency was present in cerebral blood flow velocity in 10 of 16 randomly chosen ventilated preterm infants examined at varying ages after birth. ${ }^{9}$ In view of the fact that respiratory variability has been assumed to be the major component of the possibly adverse high variability it seemed important to investigate the phenomenon systematically. This study therefore aimed to examine a cohort of very low birthweight infants for the presence of a respiratory component in blood pressure and cerebral blood flow velocity variability, and to explore which factors were important in determining the presence or absence of this variability.

\section{Patients and methods}

INSTRUMENTS

Duplex Doppler ultrasound (ATL Mk 600) was used to measure cerebral blood flow velocity in the anterior cerebral artery via the anterior fontanelle. The cerebral blood flow velocity signal was processed by a microcomputer based system developed by Schlindwein et al, which used fast Fourier transform analysis to calculate the peak velocity every 6.25 ms. $^{10}$ The result was passed to a second microcomputer, an Apple Macintosh II running Labview, where it was stored. Simultaneous recordings were taken of ventilator pressure using a pressure transducer (Mercury M11), blood pressure from the arterial line using a Sorensen intraflow transducer, and spontaneous respiratory effort from a 
Ventilator pressure

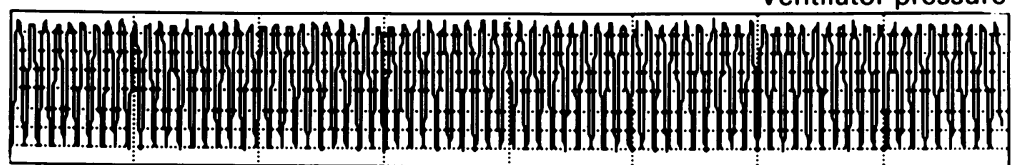

Blood pressure
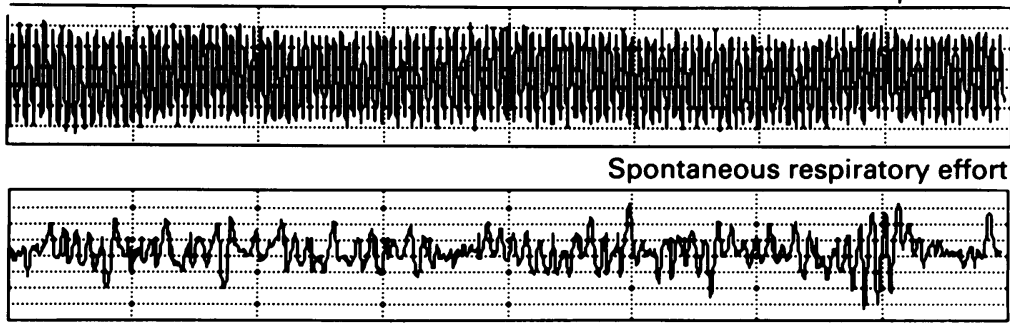

CBFV

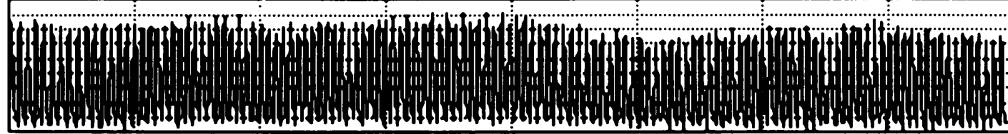

Figure 1 Example of one minute recording of four channels of physiological information; $C B F V=$ cerebral blood flow velocity.
Spontaneous respiratory effort

SIGNAL ANALYSIS

Each one minute windowed epoch of recorded signals from the ventilator pressure, blood pressure, spontaneous respiratory effort, and cerebral blood flow velocity was subjected to Fourier transform spectral analysis using Labview. A logarithmic scale was used for display (fig 2). Spectral analysis allowed the identification of two different patterns which were allocated without access to clinical information or to the name of the patient. In the first, a peak was visible at either the spontaneous or ventilator respiratory frequency in the blood pressure and the cerebral blood flow velocity traces. Another peak was always seen at the heart rate frequency (fig $2 \mathrm{~A}$ ). In the second response, no peak at either the spontaneous or ventilator frequency was seen in the cerebral blood flow velocity trace (fig 2B). A respiratory frequency was always noted in the blood pressure trace. The peaks which are seen at precise multiples of the first, for example, at 1.35 and $2.7 \mathrm{~Hz}$ on the ventilator channel of fig $2 \mathrm{~B}$, are harmonics.

respiratory monitor (Graseby MR10). The analogue outputs were sampled at $30 \mathrm{~Hz}$ and stored together with the cerebral blood flow velocity signal within Labview. Figure 1 shows an example of a four channel recording lasting one minute.

\section{PATIENTS}

Seventy three infants of birth weight less than $1500 \mathrm{~g}$ and postnatal age less than 24 hours were admitted to the special care baby unit at the Rosie Maternity Hospital in Cambridge between December 1989 and October 1990 and were all enrolled in this trial; 62 had functioning indwelling arterial catheters. Recordings were made on days $1,2,3$, and 7 and each recording lasted for one minute. Informed parental consent was obtained before the recordings were made and the study was approved by the Cambridge district ethical committee.

Independent observers assessed and recorded the appearance of each infant's cerebral ultrasound scan from a real time image made at weekly intervals.

Information on the clinical course of each infant was collected prospectively. Blood gases were checked during each study if an arterial line was present. Ventilator settings were noted. Table 1 gives some of the more relevant factors. Diagnosis of patent ductus arteriosus was made using Doppler ultrasound to assess the presence or absence of turbulent flow in the pulmonary artery. ${ }^{11}$

Table 1 Factors recorded prospectively and used in univariate and multivariate analysis

\begin{tabular}{|c|c|}
\hline Respiratory factors & $\begin{array}{l}\text { Peak pressure } \\
\text { Rate } \\
\text { Hours in oxygen } \\
\text { Percentage inspired oxygen } \\
\text { Arterial blood gas results }\end{array}$ \\
\hline Circulatory factors & $\begin{array}{l}\text { Blood pressure, inotropic support } \\
\text { Cerebral injury, patent ductus } \\
\text { Cerebral blood flow velocity } \mathrm{cm} / \mathrm{s}\end{array}$ \\
\hline General details & $\begin{array}{l}\text { Maternal illness, mode of delivery, } \\
\text { sex, gestation, birth weight, } \\
\text { sepsis, outcome }\end{array}$ \\
\hline
\end{tabular}

STATISTICAL ANALYSIS

To explore the factors which may have been associated with the different response logistic regression was used (statistical package for the social sciences (SPSS)). ${ }^{12}$ This form of statistical analysis was chosen because the dependent variable - that is, the pattern of response for each recording made-was a dichotomous categorical variable. $\chi^{2}$, Fisher's exact test, and $t$ tests were also used to compare groups before analysis with logistic regression. The SPSS file contained data on 73 infants and over 200 variables; up to six were considered with logistic regression at any one time.

\section{Results}

Two hundred and forty nine recordings were made from 73 infants. Thirty seven infants were studied on all four days, with the remainder contributing fewer epochs due mainly to early death. Eight infants had five recordings made because a separate epoch was collected at the time that a clinical diagnosis of patent ductus arteriosus was made in view of the possibility that this would influence cerebral blood flow velocity variability. Figure 3 shows the numbers of observations made on each infant. Figure 4 shows the distribution of responses over the first week of life. The presence of respiratory associated variability in cerebral blood flow velocity was seen less often as the first week of life progressed, and was most likely to occur on the first day after birth, being present at this time in 40 of 70 infants studied. Thirty seven of these infants were ventilated at this time versus 19 of those who did not show respiratory induced variability $\left(\chi^{2}=12 ; \mathrm{p}<0 \cdot 005\right)$. Even in ventilated infants, however, the frequency which was represented in the cerebral blood flow velocity was more likely to be the spontaneous respiratory frequency rather than the 

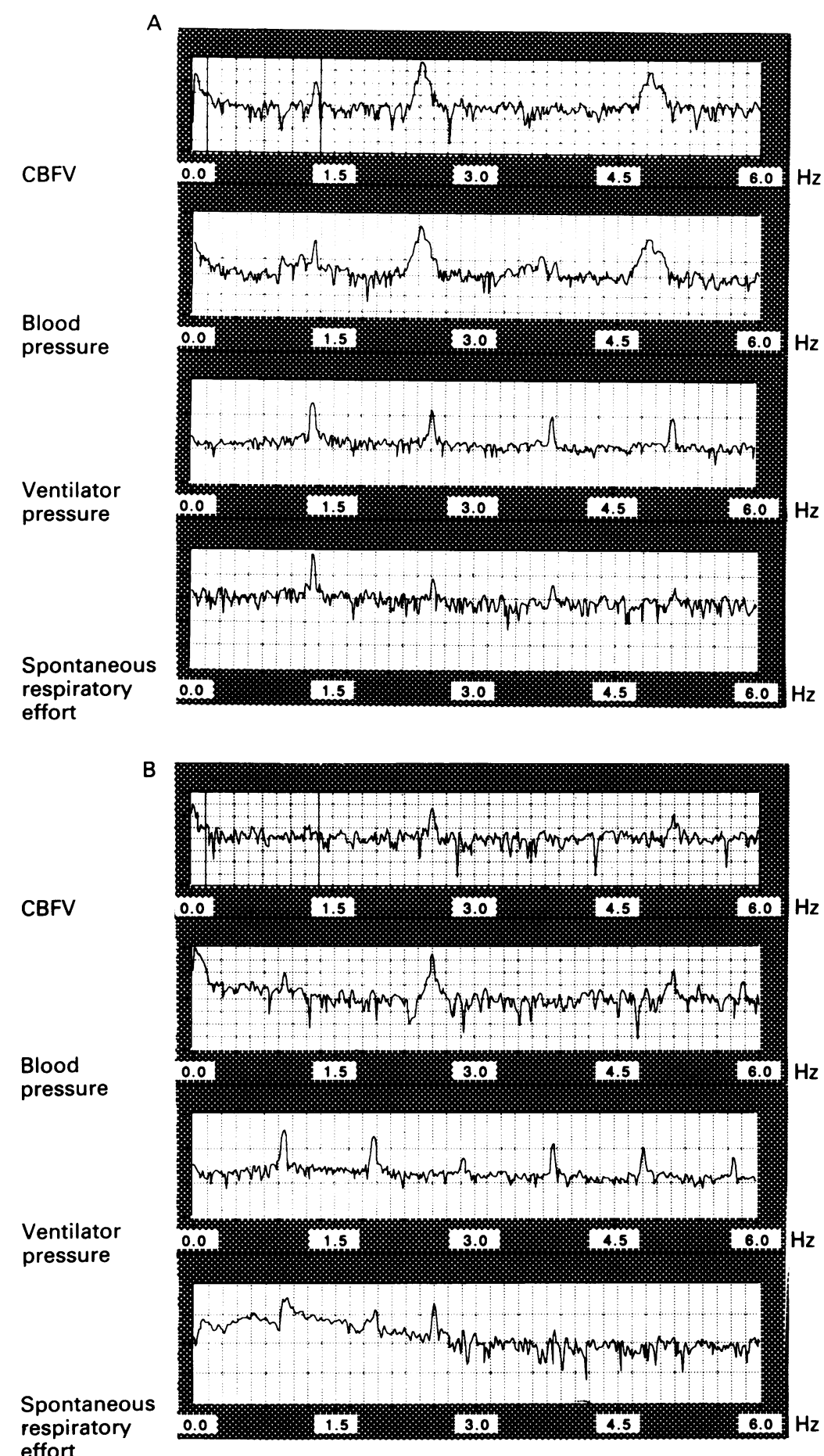
effort

Figure 2 Example of the result of a Fourier transform of the raw data. (A) showing the presence and $(B)$ the absence of respiratory associated variability in cerebral blood flow velocity $(C B F V)$.

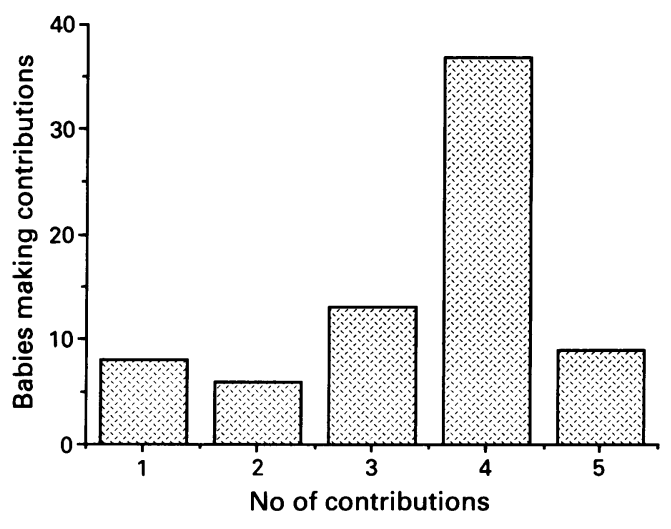

Figure 3 Number of babies studied showing the number of epochs contributed by each.

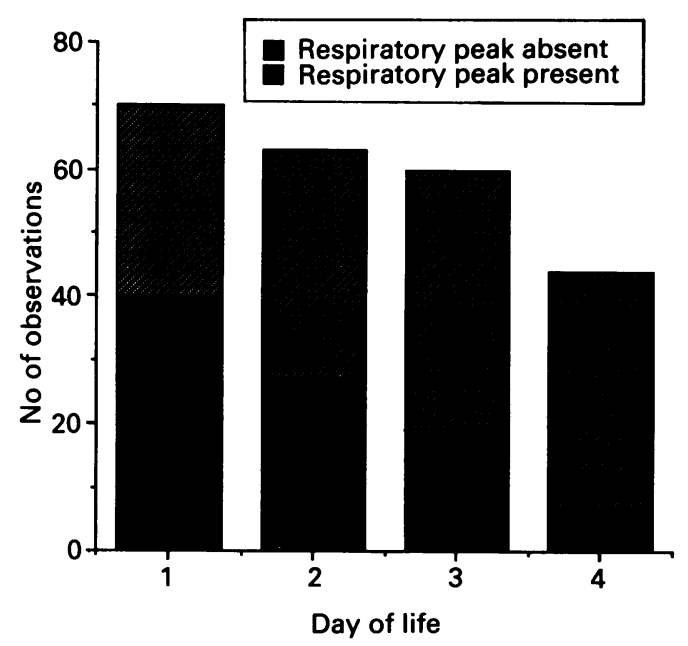

Figure 4 The distribution of respiratory associated variability over the first week of life.

time. Each infant contributed only once to this data set. Secondly, to explore the influence of ventilator settings, blood pressure, and blood gas variables during separate epochs, each recording was considered as a separate case. This allowed an analysis considering information pertaining to the situation at the time of the study to be examined, but most infants contributed more than once to this data set (fig 3). Some of the variables available for analysis, which had been collected prospectively, are shown in table 1 .

CHARACTERISTICS OF THE INFANTS SHOWING RESPIRATORY INDUCED VARIABILITY

Forty seven of the 73 infants showed the presence of a respiratory frequency in cerebral blood flow velocity at any time. In 40 infants this pattern was seen on the first day (fig 2). Univariate analysis suggested that the infants who showed respiratory associated variation were smaller, less mature, and more likely to be ventilated using a higher peak pressure, rate, and inspired oxygen concentration (table 2). They were more likely to die or sustain brain injury than those who never showed such a variation in their brain blood flow. There were more infants who were clinical course and outcome were examined, related to whether or not the infant ever showed respiratory induced variability at any 
Table 2 Factors related to the presence of respiratory variability in cerebral blood flow velocity

\begin{tabular}{|c|c|c|c|c|}
\hline \multirow[b]{3}{*}{$\begin{array}{l}\text { Mean (SD) birth weight (g) } \\
\text { Mean (SD) gestation (weeks) } \\
\text { Mean (SD) days in oxygen } \\
\text { Death } \\
\text { Intraventricular haemorrhage grade 2+ } \\
\text { Periventricular leucomalacia } \\
\text { Blood pressure }<30 \mathrm{~mm} \mathrm{Hg} \mathrm{ever}\end{array}$} & \multicolumn{3}{|c|}{ Respiratory variability } & \multirow{2}{*}{$\begin{array}{l}\text { Univariate } \\
\text { p value }\end{array}$} \\
\hline & \multicolumn{2}{|c|}{$\begin{array}{l}\text { Present } \\
(n=47)\end{array}$} & $\begin{array}{l}\text { Absent } \\
(n=26)\end{array}$ & \\
\hline & $\begin{array}{r}1088 \\
27 \\
5 \\
11 \\
10 \\
2 \\
17\end{array}$ & $\begin{array}{l}(243) \\
(1 \cdot 8) \\
(48 \cdot 3)\end{array}$ & $\begin{array}{cc}1259 & (227) \\
29 \cdot 4 & (2 \cdot 4) \\
1 & (22 \cdot 2) \\
0 & \\
0 & \\
1 & \\
4 & \end{array}$ & $\begin{array}{l}0.004 \\
0.002 \\
0.04 \\
0.007 \\
0.01 \\
\text { NS } \\
0.05\end{array}$ \\
\hline
\end{tabular}

hypotensive at any time in the respiratory associated variability group $(p=0 \cdot 05)$. Logistic regression analysis showed that gestational age was the most significant factor $(p=0.0018)$, and that when the other factors were considered together with gestational age they did not have a significant independent effect.

\section{FACTORS RELATED TO THE EPOCH WHEN} RESPIRATORY INDUCED VARIABILITY WAS NOTED Two hundred and forty nine epochs were available for analysis. Ninety seven showed the presence of a respiratory frequency in cerebral blood flow velocity. Univariate analysis (table 3 ) suggested once more that factors relating to artificial ventilation were the most important; blood gas results were not implicated, nor was the type of sedation used. Hypotension during the study epoch was significant as were the total hours of hypotension recorded during the day on which the study was made. Logistic regression analysis showed that whether the infant was ventilated or not was the most significant factor, doubling the odds of the infant being in the group with any respiratory frequency represented in the cerebral circulation. The effect of ventilator rate was wholly accounted for by whether or not artificial ventilation was used; in other words, there was no independent effect of rate. The inspired oxygen concentration also had a highly significant independent effect on the type of response; for every additional $10 \%$ increase in inspired oxygen there was an increase in the odds of a respiratory frequency appearing in the cerebral blood flow velocity of $1 \cdot 4: 1$. The effects of ventilation and oxygen were not accounted for by birth weight or gestational age. Hypotension was not shown to have an independent effect.

Table 3 Significant factors related to the presence of respiratory variability in cerebral blood flow velocity by epoch

\begin{tabular}{|c|c|c|c|c|c|}
\hline & \multicolumn{4}{|c|}{ Respiratory variability } & \multirow{2}{*}{$\begin{array}{l}\text { Univariate } \\
\text { p value }\end{array}$} \\
\hline & \multicolumn{2}{|c|}{$\begin{array}{l}\text { Present } \\
(n=97)\end{array}$} & \multicolumn{2}{|c|}{$\begin{array}{l}\text { Absent } \\
(n=152)\end{array}$} & \\
\hline $\begin{array}{l}\text { Blood pressure }<30 \mathrm{~mm} \mathrm{Hg} \text { ever } \\
\text { No of hours blood pressure }<30 \text { in } 24 \\
\text { CBFV }(\mathrm{cm} / \mathrm{s}) \\
\text { Peak ventilator pressure }\left(\mathrm{cm} \mathrm{H}_{2} \mathrm{O}\right) \\
\text { Inspired oxygen }(\%) \\
\text { Ventilator rate } \\
\text { Ventilated epochs } \\
\text { Synchronous ventilation }\end{array}$ & $\begin{array}{l}8 \\
1 \cdot 41 \\
9 \cdot 9 \\
16 \\
50 \\
65 \\
72 \\
64\end{array}$ & $\begin{array}{l} \pm 3 \cdot 8 \\
(7 \cdot 7-12 \cdot 9) \\
(14 \cdot 5-21 \cdot 5) \\
(32-70) \\
(30-80)\end{array}$ & $\begin{array}{l}0 \\
0 \cdot 16 \\
11 \\
0 \\
21 \\
0 \\
55 \\
42\end{array}$ & $\begin{array}{l} \pm 0 \cdot 8 \\
(7 \cdot 8-15 \cdot 2) \\
(0-16) \\
(21-30) \\
(0-20)\end{array}$ & $\begin{array}{l}<0.001 \\
<0.002 \\
0.2 \\
<0.0001 \\
<0.0002 \\
<0.0001 \\
<0.0001 \\
0.05\end{array}$ \\
\hline
\end{tabular}

Interquartile ranges in brackets.

$\mathrm{CBFV}=$ cerebral blood flow velocity.

\section{Discussion}

This study has confirmed, in a cohort of very low birthweight infants, that 'pulsus paradoxus' was present in the cerebral circulation at some time in more than half of the infants. Pulsus paradoxus is usually recognised as a variation of blood pressure occurring at the same frequency of respiration and is a normal phenomenon when the direction of change is such that there is a small decrease in blood pressure with inspiration and the magnitude of the change is less than $10 \mathrm{~mm} \mathrm{Hg}$ in adults. It is thought to be due to a central modulation of the baroreceptor reflex either by a direct neural communication from the respiratory centre or via thoracic volume receptors. The same phenomenon is responsible for respiratory sinus arrhythmia, and the amplitude of both these changes is increased when respiration occurs at or near to the natural oscillating frequency of the baroreceptors (about $0.1 \mathrm{~Hz}$ in adults). Respiratory associated variability was more likely to occur in the cerebral circulation of those infants of short gestation on the first day of life, and independently of gestational age in those who were ventilated in high oxygen concentrations. These findings perhaps explain the previous link suggested between high beat to beat variability and cerebral injury. ${ }^{1}$ The association with cerebral injury in this group was striking, with 12 of the 13 infants with cerebral injury in the group showing respiratory associated variability, but the association was not independent of gestational age.

Previous work has shown that respiratory sinus arrhythmia exists in the heart rate of newborn infants, albeit with a lower power than in adults. ${ }^{13}$ The smaller effect of respiration on the heart rate in newborn infants is due to the larger difference between the spontaneous respiratory frequency $(0.4 \mathrm{~Hz})$ and the natural baroreceptor oscillation frequency $(0.1 \mathrm{~Hz}$ in adults), making entrainment less likely than in adults where the two frequencies are closer. Giddens and Kitney also described a computer model of sinus arrhythmia and showed that a longer delay time of $3.3 \mathrm{sec}-$ onds was necessary to stimulate the neonatal condition than that needed for adults (two seconds). ${ }^{13}$ The observations seen in the present study therefore seem unlikely to represent solely the respiratory entrainment of baroreceptor activity as the frequency at which respiration was occurring was higher than that usually considered to be the natural baroreceptor oscillating frequency. It may be, however, that artificial ventilation at a similar frequency to natural respiration was sufficient to cause entrainment of a central oscillator or to set up an influence of their own. This explanation does not require that a failure of autoregulation existed allowing direct pressure transmission of changes in aortic blood pressure to the cerebral vessels. The independent effect of oxygen is probably due to an association with the severity of respiratory distress syndrome, though infants with stiff lungs have been shown to transmit less ventilator pressure to the periphery of the lung ${ }^{14}$ so that 
an alternative explanation, perhaps invoking the effect of circulating catecholamines or oxygen itself, is required. Examination of the phase relation of the changes in the systemic and cerebral circulations and the heart rate changes will elucidate this further and is now in progress.

It is now clear that there are several components to the variability seen in cerebral blood flow velocity and that respiratory associated variability undoubtedly exists, particularly in infants of low gestational age ventilated in high oxygen concentrations on the first day of life. The association with hypotension and cerebral injury which was apparent on univariate analysis and supports previous work from ourselves $^{15}$ and others ${ }^{1}$ did not stand up to logistic regression analysis in this study and appeared to be wholly accounted for by other variables. There may be a danger of misinterpretation because of the association of variables examined with regression analysis, but it seems unlikely that gestation, brain injury, and hypotension are completely colinear (all infants of low gestational age are not brain injured) and from a statistical viewpoint the results suggest that gestational age was the most important factor. The phenomenon of respiratory variability remains interesting, but may be less harmful than was previously supposed.

We thank all the parents who gave permission for their infants to be studied, the nursing staff for their patience, and Drs to be studied, the nursing staff for their patience, and Drs
Colin Morley and Cliff Roberton for allowing their patients to be included. Our future studies in this area will be supported by Action Research.

1 Perlman JM, McMenamin JB, Volpe J. Fluctuating cerebral blood-flow velocity in respiratory-distress syndrome. N Engl f Med 1983;309:204-9.

2 Cowan F, Thoresen $M$. Effects of intermittent positive pressure ventilation on cerebral arterial and venous velocities in newborn infants. Acta Paediatr Scand 1987; 76:239-47.

3 Rennie JM, South M, Morley CJ. Cerebral blood flow velocity variability during assisted ventilation in premature babies. Arch Dis Child 1987;62:1247-51.

4 Hales S. Statistical essays. Vol 2. London: Innes, 1733; cited in Dornhurst AC, et al. Respiratory variations in cited in Dornhurst AC, et al. Respirator
blood pressure. Circulation 1952;7:558.

5 Traube L. Uber periodische Tatigkeitsanderungen des vasomotorischen und Hemmings-Nervenzentrums. Cbl Med Wiss 1865;56:88-5.

6 Hering E. Uber den Einfluss der Atmung auf den Kreislauf. Sber Akad Wiss Wien 1869;60:829-36.

7 Kitney RI. New findings in the analysis of heart rate variability in infants. Automedica 1984;5:289-310.

8 Hyndman BW, Kitney RI, Sayers BMc. Spontaneous rhythms in physiological control systems. Nature 1971; 233:339-40.

9 Bignall S, Bailey PC, Rivers RPS, Lissauer TJ. Quantification of cardiovascular instability in premature infants using spectral analysis of waveforms. Pediatr Res 1988;23:398-401.

10 Schlindwein FS, Smith MJ, Evans DH. Spectral analysis of Doppler signals and computation of the normalised first moment in real time using a digital signal processor. Med Biol Eng Comput 1988;26:228-32.

11 Kupferschmid Ch, Lang D, Pohlandt F. Sensitivity, specificity and predictive value of clincal findings, m-mode echocardiography and continuous-wave Doppler sonography in the diagnosis of symptomatic patent ductus arteriosus in preterm infants. Eur $\mathcal{F}$ Pediatr 1988;147: 279-82.

12 Norusis MJ. SPSS- $X$ user's guide for the Macintosh. Chicago: SPSS-X Inc, 1990

13 Giddens DP, Kitney RI. Neonatal heart rate variability and its relation to respiration. $\mathcal{F}$ Theor Biol 1985;113:759-80.

14 Greenough A, Morley CJ. Oesophageal pressure measurements in ventilated preterm babies. Arch Dis Child 1982;57:851.

15 Rennie JM. Cerebral blood flow velocity following cardiovascular support in premature babies. Arch Dis Child 1989;64:897-90. 